\title{
Phase transition in a spatial Lotka-Volterra model
}

\author{
György Szabó ${ }^{1}$ and Tamás Czárán ${ }^{2,3}$ \\ ${ }^{1}$ Research Institute for Technical Physics and Materials Science \\ POB 49, H-1525 Budapest, Hungary \\ ${ }^{2}$ Theoretical Biology and Ecology Research Group of the Hungarian Academy of Sciences \\ ${ }^{3}$ Department of Plant Taxonomy and Ecology, Eötvös University \\ H-1083 Budapest, Ludovika tér 2, Hungary \\ November 1, 2018
}

\begin{abstract}
Spatial evolution is investigated in a simulated system of nine competing and mutating bacterium strains, which mimics the biochemical war among bacteria capable of producing two different bacteriocins (toxins) at most. Random sequential dynamics on a square lattice is governed by very symmetrical transition rules for neighborhood invasions of sensitive strains by killers, killers by resistants, and resistants by sensitives. The community of the nine possible toxicity/resistance types undergoes a critical phase transition as the uniform transmutation rates between the types decreases below a critical value $P_{c}$ above which all the nine types of strain coexist with equal frequencies. Passing the critical mutation rate from above, the system collapses into one of three topologically identical (degenerated) states, each consisting of three strain types. Of the three possible final states each accrues with equal probability and all three maintain themselves in a self-organizing polydomain structure via cyclic invasions. Our Monte Carlo simulations support that this symmetry-breaking transition belongs to the universality class of the three-state Potts model.
\end{abstract}

PACS numbers: 87.23.Cc, 05.10.-a, 05.40.Fb, 64.60.Ht

Many species of bacteria have recently been shown to excrete toxic subtances that are very effective against strains of the same or closely related species not producing the corresponding resistance factor [1.2]. With respect to a certain toxin a species consists of colonies of three possible types: Sensitive $(S)$, Killer $(K)$, or Resistant $(R)$. Killer strains produce the toxin and a resistance factor that prevents suicide; resistant strains only produce the resistance factor, and sensitives produce neither. An $S$ colony can always be invaded and displaced by a $K$ propagulum because $K$ can kill $S$ using the toxin. A $K$ colony can be invaded and ultimately displaced by an $R$ propagulum because the resistant type is immune to the toxic effect and it does not carry the metabolic burden of synthetizing the toxin, thus it achieves a higher growth rate and competitive dominance over $K$. The sensitive $(S)$ type can in turn displace the resistant by competition, for it does not even pay the metabolic cost of producing the resistance factor. The resulting cyclic pattern of competitive dominance ( $K$ beats $S$ beats $R$ beats $K$ ) is a striking realization of the well-known RockScissors-Paper game [3], by a biological entity. Other cyclic dominance systems are almost unknown in ecology, but given the extraordinary significance of bacterial communities in virtually all ecological systems, the problem is well worth detailed theoretical studies.

Some theoretical aspects of cyclic dominance have already been thoroughly investigated [4, 3. In the simplest spatial (lattice) version of a cyclic Lotka-Volterra system [5.6] the species are distributed on a $d$-dimensional lattice, and invasions are confined to nearest neighbor sites with uniform rates. [7,8] Analytical and numerical calculations have proven that fixation occurs if the number of species exceeds a critical value dependent on dimension $d$, otherwise a self-organizing domain structure is maintained for $d \geq 2$. [9], which comprises rotating vortices and antivortices in three-species models. [7, 10] The present work is meant to demonstrate that extending the cyclic dominance approach to a two-toxin bacterial community with mutation results in a remarkable enrichment of interesting dynamical phenomena, compared to what is already known.

The relevant biological details of bacteriocin systems are the following: The genes coding for the toxin and the resistance factor are usually both sitting on an extrachromosomal DNA-ring in the citoplasm (called plasmid) that the bacterium can lose and obtain without any immediate deleterious impact. Each of the two genes on the plasmid can be switched off by DNA mutation. Thus all possible mutational transformations are possible in principle, but - supposing the mutant does not disperse far immediately - those having a visible effect are only the ones after which the mutant defeats the resident strain from which it emerged. Obviously, $S \rightarrow K, K \rightarrow R$, and $R \rightarrow S$ are mutations followed by competitive displacement of the resident, i.e., they are permitted, but the reverse ones are immediately eliminated by the resident population. $S \rightarrow K$ involves obtaining a complete plasmid which is possible, e.g., through genetic transformation or a sexual event called conjugation; the other two viable mutations are realized by switching off the toxin gene and the resistance gene, respectively, on an existing plasmid.

Most bacteria are capable of producing more than one toxin and/or the corresponding resistance factors simultaneously. If the maximum number of toxin types is two, 
the number of possible toxicity/resistance combinations in a strain is nine. These are: $S S, S K, S R, K S, K K$, $K R, R S, R K$, and $R R$. Here we confine our attention to this two-toxin system, denoting the actual state of a bacterium colony by an index number from 0 to 8 in the order above. The topology of the dominance relations among the states is illustrated in Fig. 11. The biological justification for this topology is straightforward: double dominance of strain A above strain B means that A harbors dominant genes on both plasmids compared to B; single dominance means that one gene of $\mathrm{A}$ is dominant, the other is identical to that of the correspondig gene in $\mathrm{B}$; no dominance follows either if the corresponding genes are both identical, or if the two genes play draw (i.e., A wins with one gene, and $\mathrm{B}$ with the other).

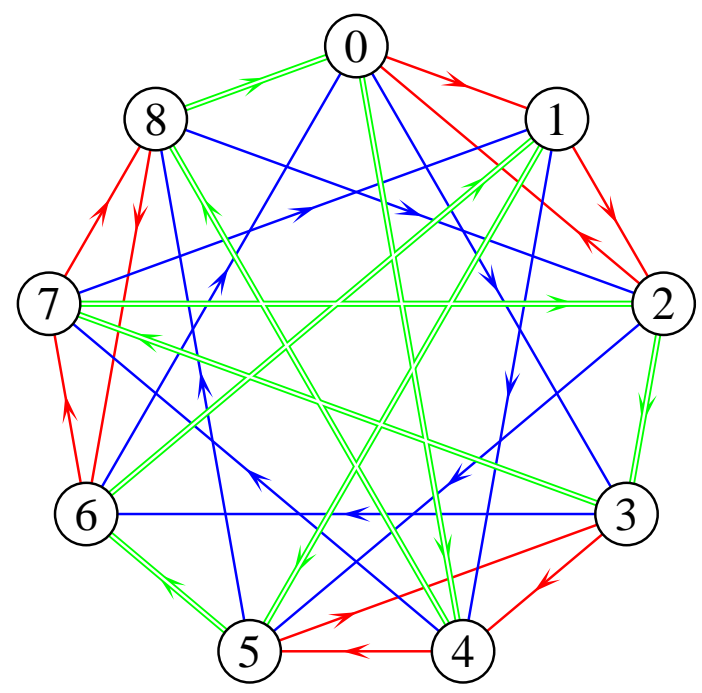

FIG. 1. Topology of dominance in the nine-species model. The single and double lines with arrow indicate single and double dominances as described in the text.

We use a square lattice of size $L \times L$ with periodic boundary conditions as the arena for interaction. Each lattice site $i$ is occupied by a colony of one of the nine toxicity/resistance types (we call them "species" for brevity in the sequel). Assignment of a certain value of the state variable $s_{i}=0, \ldots, 8$ to a certain site indicates the presence of the corresponding species on that site. The simulation works by iterating the following elementary steps: At a randomly chosen site one of the two possible mutants replaces the resident colony with probability $P$, otherwise the resident colony fights with a randomly chosen nearest neighbor colony by mutually invading propagules (with a probability $1-2 P)$. The outcome of the battle between two neighbors ( $i$ and $j$ ) depends on the dominance relations between them: $i$ displaces $j$ and takes over its site if $s_{i}$ is dominant over $s_{j}$ (cf. Fig. 1). If the neighbors are equivalent or neutral to each other (play draw), nothing happens.

Lattice size varies from $L=400$ to 3000 in the simulations. At $t=0$ the species were distributed at random with uniform probabilities on the lattice in all simulation runs. The control parameter was mutation rate, varying from 0 to $1 / 2$. We have recorded the time series of species concentrations and correlation functions, the latter of which served as the basis for calculating correlation length. After a suitable thermalization time we have averaged these data over some sampling time chosen to be long enough for providing sufficient accuracy in spite of the occassionally very high concentration fluctuations.

In no-mutation runs $(P=0)$ we have observed an interesting domain size increase phenomenon in the time series of spatial patterns which develop. One can distinguish three equivalent types of growing domains consisting of the $0+4+8,1+5+6$, and $2+3+7$ species respectively. Inside these three domains a self-organizing structure is maintained through the mechanism described by Tainaka for the simplest 3-species cyclic dominance model [7]. We call these domains "alliances" henceforth, but note that the species within an alliance are in fact the worst enemies: each alliance consists of species cyclically double-dominating each other. Our reason for this terminology will be clear in a minute.

The three alliances are given approximately equal territories at start, but the system slowly drifts towards a single-domain state in all simulations. Each alliance has the same chance to take over.

Alliances defend themselves against the external invasion of "alien" species with a peculiar mechanism. One can easily check that any external invador can attack only one of the species within an alliance, and the invador is eliminated from the domain of the alliance by the species actually controlling the attacked one within the alliance. This means that the invador is wiped away by the toughest within-domain enemy of the attacked species, thus maintaining the self-organizing structure and the integrity of the domain with the very same mechanism.

The self-protection of alliances against external invadors can also be observed for small mutation rates as illustrated in Fig. 2. In this snapshot similar symbols are used for species belonging to the same alliance. Namely, different strip widths (or box sizes) distinct the species within the three alliances represented by horizontal and vertical strips and closed squares respectively. This figure illustrates that the mutants and their offspring can form only small, temporary islands in the sea of the dominant domain $(0+4+8)$ represented by vertical strips. Clearly, the concentrations of minority species increase with $\mathrm{mu}-$ tation rate $P$.

The average concentrations of the species become equal if the mutation rate exceeds a critical value $P_{c}$. This continuous transition is accompanied by a divergence in both the fluctuations and the correlation length. A similar phase transition occurs in the three-state Potts model [11,12, therefore we have adopted the numerical techniques suggested by Binder [13] for the quantitative analyses. 


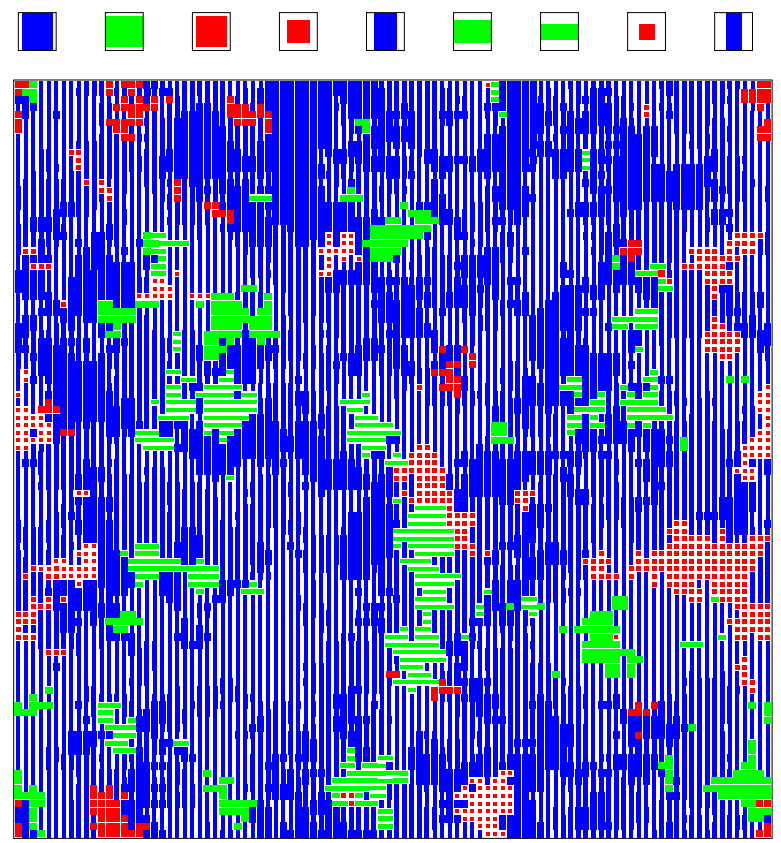

FIG. 2. Snapshot of species pattern for $P=0.0003$. The different symbols magnified at the top represent the species $s=0, \ldots, 8$ from left to right.

In order to reduce relaxation time in systematic investigations, the random initial state contained only species 0,4 , and 8 at small values of $P$. The MC simulations were performed on large lattices $(L>1500)$ and long sampling times $\left(t>3 \cdot 10^{5} \mathrm{MC}\right.$ steps per sites $)$ in the vicinity of the critical point. For such large lattice sizes the dominance of the $0+4+8$ alliance was maintained during the simulations at all tested values of $P$ below $P_{c}$.

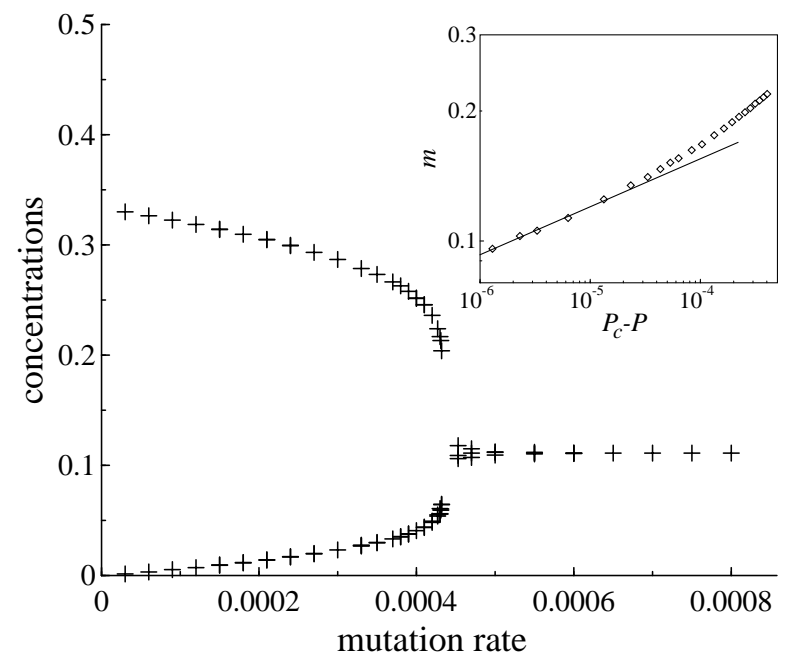

FIG. 3. Average concentrations of species as a function of mutation rate. The inset shows the log-log plot of the order parameter vs. $P_{c}-P$.

The simulations suggest a continuous transition as in- dicated in Fig. 3. Statistical errors are small, comparable to the line thickness of the figure, except for the very close vicinity of the critical point.

Figure 3 shows that the $P$-dependence of average concentrations can be characterized by a single order parameter $m$ as

$$
\begin{aligned}
& c_{0}=c_{4}=c_{8}=\frac{1}{9}+m, \\
& c_{1}=c_{2}=c_{3}=c_{5}=c_{6}=c_{7}=\frac{1}{9}-\frac{1}{2} m .
\end{aligned}
$$

According to our MC simulations, $m$ follows a power law behavior in the close vicinity of $P_{c}$, i.e.

$$
m \propto\left(P_{c}-P\right)^{\beta}
$$

if $P<P_{c}$ (see the inset in Fig. 3), whereas the order parameter remains zero for $P>P_{c}$. Numerical fitting yields $\beta=0.110(5)$ and $P_{c}=0.0004333(5)$. This value of the $\beta$ exponent is in good agreement with the theoretical prediction $(\beta=1 / 9)$ obtained for the three-state $(Q=3)$ Potts model 12. This is not surprising, given that a large class of two-state dynamical systems exhibits phase transition belonging to the universality class of the Ising model [14] and the $Q$-state Potts model was introduced as a generalization of the Ising model [11,12].

To obtain further evidence, we have also studied some other quantities characterizing the critical behavior. For example, the fluctuation of the order parameter defined as $\chi=N\left\langle(m-\langle m\rangle)^{2}\right\rangle$ can be well approximated by a power law; $\chi \approx\left|P-P_{c}\right|^{\gamma}$ in the vicinity of $P_{c}$. Below and above the critical point, numerical fitting yields $\gamma=1.3(2)$ and $\gamma^{\prime}=1.43(4)$ respectively, which values agree with the theoretical prediction $\left(\gamma=\gamma^{\prime}=13 / 9\right)$ [12. The investigation of the cumulant of the order parameter 15] for small lattice sizes $(L=60,100,200)$ supports the presence of a continuous transition at the critical mutation rate $P_{c}$. Furthermore, we have determined the correlation function $C(x)$ for $P>P_{c}$, which characterizes the probability of finding the same species on two sites at a distance $x$ away from each other. In the vicinity of $P_{c}$, two different characteristic lengths can be obtained from $C(x)$ (see the inset in Fig. 14). The shortest correlation length is proportional to the linear size of a domain within the alliance, and this quantity remains finite if $P \rightarrow P_{c}$. The longest correlation length is more interesting, because it characterizes the linear size of the alliance and diverges if $P \rightarrow P_{c}$. More precisely, this correlation length can be well described by a power law $\left(\xi \sim\left(P-P_{c}\right)^{\nu}\right)$ as illustrated in Fig. 4 . Numerical fitting predicts $\nu=0.82(4)$, in agreement with the theoretical prediction $\nu=5 / 6$ for the two-dimensional, three-state Potts model [12]. 


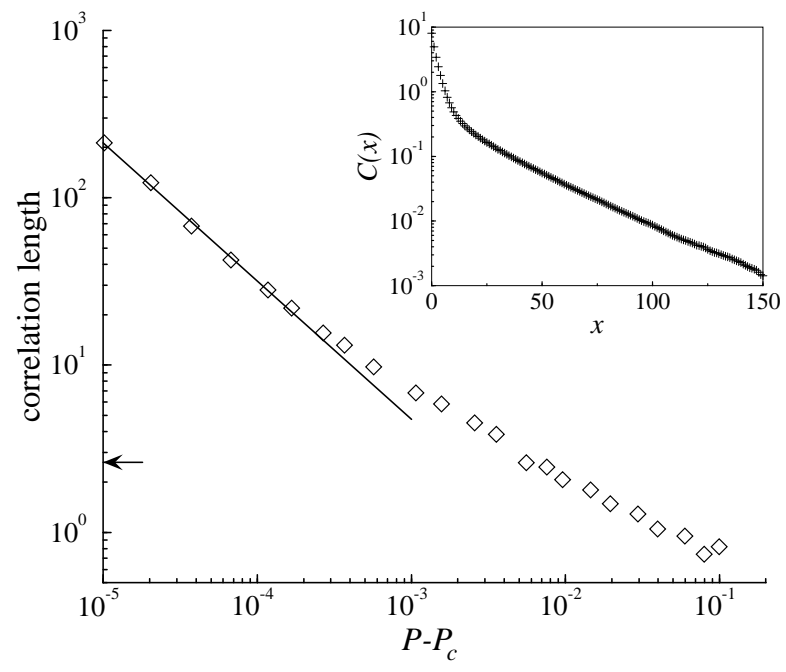

FIG. 4. Log-log plot of the correlation length characteristic to the linear extension of alliances as a function of $P-P_{c}$. The solid line represents the fitted power law behavior with a slope of -0.82 . The arrow points to the value of the correlation length characteristic to the linear domain size inside an alliance for $P=0$. The inset shows the lin-log plot of the correlation function for $P=0.00055$.

We have also investigated the model analytically, by evaluating the probability of configurations on two adjacent sites (pair approximation method). Even though the number of possible pair configurations is large $\left(9^{2}\right)$, considering the (translation, rotation, reflection, and cyclic) symmetries of the system the number of different pair probabilities reduces to as few as four. For sufficiently large $P$, this method gives a good approximation for the behavior of the simulation model (i.e., it predicts vanishing correlations if $P \rightarrow 1 / 2$ ). However, it shows no sign of the phase transition found in the MC simulations at $P_{c}$. This failure of the pair approximation method in showing the phase transition is related to the key role that interfacial invasion plays in the development of the self-organizing domain structure. [7]10] This feature limits the techniques we can use for further investigations.

In conclusion, our MC simulations justify that the present model exhibits a critical phase transition accompanied with spontaneous symmetry-breaking, in close analogy to the well-known Potts model. Here the mutation rate $P$ plays the role of the control parameter whose increase drives the system towards the symmetric stationary state in which all the nine species are present with the same probability. Conversely, for low mutation rate $P$ the system drifts towards the dominance of an alliance consisting of three species whose survival is maintained by cyclic within-domain invasion. Due to the very symmetric topology of species dominance relations the system admits three equivalent alliances. Numerical analysis of the critical behavior $(\beta, \nu$, and $\gamma$ exponents) supports our conjecture that the observed phase transition belongs to the universality class of the three- state Potts model. The most surprising result of this work is that cyclic invasion is capable of providing protection (stability) for alliances consisting of mortal enemies (species double-dominating each other) under some particular conditions hidden in the topology of the interaction. Further systematic research is required to clarify the conditions for the emergence of such defensive alliances accompanied by a reduction in the number of surviving species for more general interaction topologies.

\section{ACKNOWLEDGMENTS}

Support from the Hungarian National Research Fund (T-23552 and T-25793) is acknowledged.

[1] P. Reeves, The Bacteriocins (Springer Verlag, New York, 1972).

[2] R. James, C. Lazdunski, and F. Pattus, (eds) Bacteriocins, Microcins and Lantibiotics (Springer Verlag, New York, 1991).

[3] J. Hofbauer and K. Sigmund, Evolutionary Games and Population Dynamics (Cambridge University Press, Cambridge, 1998).

[4] M. Bramson and D. Griffeath, Ann. Prob. 17, 26 (1989).

[5] A. J. Lotka, Proc. Natl. Acad. Sci. USA 6, 410 (1920).

[6] V. Volterra, Lecon sur la Theorie Mathematique de la Lutte pour la Vie (Gouthier-Villars, Paris, 1931).

[7] K. Tainaka, Phys. Rev. E 50, 3401 (1994).

[8] L. Frachebourg, P. L. Krapivsky, and E. Ben-Naim, Phys. Rev. E 54, 6186 (1996).

[9] L. Frachebourg and P. L. Krapivsky, J. Phys. A: Math. Gen. 31, L287 (1998).

[10] G. Szabó, M. A. Santos, and J. F. F. Mendes, Phys. Rev. E 60, 3776 (1999).

[11] R. B. Potts, Proc. Camb. Phil. Soc. 49, 106 (1952).

[12] F. Y. Wu, Rev. Mod. Phys. 54, 235 (1982).

[13] K. Binder, J. Stat. Phys. 24, 69 (1981).

[14] G. Grinstein, C. Jayaprakash, and Yu He, Phys. Rev. Lett. 55, 2527 (1985).

[15] K. Binder and D. W. Heermann, Monte Carlo Simulation in Statistical Physics (Springer-Verlag, Berlin, 1988). 